\title{
Ionospheric Correction of SAR Interferograms by Multiple-Aperture Interferometry
}

\author{
Hyung-Sup Jung, Member, IEEE, Dong-Taek Lee, Student Member, IEEE, Zhong Lu, Senior Member, IEEE, \\ and Joong-Sun Won, Senior Member, IEEE
}

\begin{abstract}
Interferometric synthetic aperture radar (InSAR) is a powerful technique that precisely measures surface deformations at a fine spatial resolution over a large area. However, the accuracy of this technique is sometimes compromised by ionospheric path delays on radar signals, particularly with L- and P-band SAR systems. To avoid ionospheric effects from being misinterpreted as ground displacement, it is necessary to detect and correct their contributions to interferograms. In this paper, we propose an efficient method for ionospheric measurement and correction and validate its theoretical and experimental performance. The proposed method exploits the linear relationship between the multiple-aperture interferometry phase and the azimuth derivative of the ionospheric phase. Theoretical analysis shows that a total electron content (TEC) accuracy of less than $1.0 \times 10^{-4}$ TEC units can be achieved when more than 100 neighboring samples can be averaged (multilooked), and the coherence is 0.5 . The regression analysis between the interferometric phase and the topographic height shows that the root-mean-square error can be improved by a factor of two after ionospheric correction. A 2-D Fourier spectral analysis indicates that the ionospheric wave pattern in the uncorrected power spectrum has disappeared in the power spectrum of the corrected interferogram. These results demonstrate that the proposed method can effectively remove ionospheric artifacts from an ionosphere-distorted InSAR image. Note that the method assumes that there is no appreciable surface displacement in the along-track dimension of the interferogram.
\end{abstract}

Index Terms-Advanced Land Observation Satellite (ALOS) Phased-Array-type L-band Synthetic Aperture Radar (PALSAR), interferometric synthetic aperture radar (InSAR), ionospheric correction, ionospheric phase map, multiple-aperture interferometry (MAI), synthetic aperture radar (SAR).

Manuscript received May 1, 2012; revised July 9, 2012; accepted September 7, 2012. Date of publication November 16, 2012; date of current version April 18, 2013. This work was supported in part by the Space Core Technology Development Program through the National Research Foundation of Korea funded by the Ministry of Education, Science and Technology under Grant 2012M1A3A3A02033465 and in part by the State-of-the-Art Remote Sensing Technology Development of Disaster Management Research Program funded by the National Disaster Management Institute under Grant NDMI-M-2012-09.

H.-S. Jung is with the Department of Geoinfomatics, The University of Seoul, Seoul 130-743, Korea (e-mail: hsjung@uos.ac.kr).

D.-T. Lee was with the Department of Geoinfomatics, The University of Seoul, Seoul 130-743, Korea. He is now with the Division of Geospatial Imagery Information and Photogrammetry, National Geographic Information Institute, Suwon 443-772, Korea (e-mail: leedt@ korea.kr).

Z. Lu is with the Cascades Volcano Observatory, U.S. Geological Survey, Vancouver, WA 98683 USA (e-mail: lu@usgs.gov).

J.-S. Won is with the Department of Earth System Sciences, Yonsei University, Seoul 120-749, Korea (e-mail: jswon@yonsei.ac.kr).

Color versions of one or more of the figures in this paper are available online at http://ieeexplore.ieee.org.

Digital Object Identifier 10.1109/TGRS.2012.2218660

\section{INTRODUCTION}

$\mathbf{S}$ YNTHETIC aperture radar (SAR) interferometry is a powerful technique that precisely measures surface deformations over a large area of thousands of square kilometers. It has been successfully used to study earthquakes [1], [2], volcanic eruptions [3], [4], glacier movement [5], [6], ground subsidence [7], [8], water-level changes in wetlands [9], etc. However, the accuracy of this technique is compromised by tropospheric and ionospheric path delays on radar signals. Because both effects cause phase distortions in radar images, SAR interferograms can contain topography and/or ground deformation signals mixed with tropospheric and ionospheric artifacts [13], [28], [29]. For the ionosphere, phase distortion depends on the ionospheric total electron content (TEC) and the inverse of the radar frequency [10]-[12]. L-band (24-cm wavelength) SAR systems such as the Advanced Land Observation Satellite (ALOS) Phased-Array-type L-band Synthetic Aperture Radar (PALSAR) can suffer from more severe ionospheric phase distortions than the X- and C-band SARs [13], [14]. To avoid ionospheric effects being misinterpreted as ground displacement, it is necessary to detect and correct their contribution to interferograms.

The ionosphere's influence on interferometric phase has recently been well studied, and several remediation methods have been proposed. These can be divided into three main categories: 1) range split-spectrum method based on the difference of the path delay between the two observations from different wavelengths [14], [19]-[21]; 2) range group-phase delay difference method [11], [14]; and 3) azimuth shift method based on the relationship between the azimuth derivative of the ionospheric effect and the azimuth displacement [11], [12]. The ionospheric influence on polarization through Faraday rotation is not considered in this paper because its effects on interferometric phase can be ignored, particularly for the L-band SAR system [15]-[18].

The range split-spectrum method exploits the dispersive nature of radar signals to estimate the ionospheric contribution [14], [19]-[21]. While topography, ground deformation, and atmospheric artifacts are nondispersive, the ionospheric effect is dispersive. Thus, the ionospheric effect can be separated by observations at two different frequencies. This method can theoretically be useful for interferometric SAR (InSAR) ionospheric corrections because it has been successfully used for correcting the ionospheric contribution in GPS measurements. However, the accuracy of the method for the required resolutions is limited for the current SAR system due to its low range bandwidth (14 or $28 \mathrm{MHz}$ ) [14].

The range group-phase delay difference method exploits the differences in sign between the range group and phase delays 
caused by the ionosphere [11], [14]. This method estimates the ionospheric effect by subtracting the range phase delay from the range group delay. The range phase delay is calculated from the unwrapped interferogram, while the range group delay is obtained by estimating the local range displacement through a correlation technique. This method is very time consuming because it requires highly accurate coregistration and is not good enough to correct the ionospheric effect from the L-band ALOS PALSAR interferogram due to lower range resolution [11], [14].

The azimuth shift method uses the rationale that the azimuth derivative of the ionospheric phase distortion on radar interferogram is linearly proportional to the azimuth displacement [11], [12]. This method has been derived in [11] and implemented in [12]. It calculates the azimuth derivative of the ionospheric phase distortion from the azimuth displacement and then estimates the phase distortion through azimuth integration. The potential of this method to correct the ionospheric effect is great because the estimation of the azimuth derivative of the ionosphere effect can be obtained at a higher spatial resolution [11]. However, from the perspective of practical implementation, the integral constant for integrating azimuthal ionospheric gradients has not been introduced, and a clear validation of the ionospheric correction has not been carried out. Moreover, this method is time consuming and has reduced sensitivity because the offset tracking method is used in the determination of azimuth offsets [22]-[24].

In this paper, we propose an efficient method utilizing multiple-aperture interferometry (MAI) to measure and correct ionospheric phase distortion by improving the azimuth shift method. We also evaluate the theoretical and experimental performances of the proposed method. The innovation of the proposed method is to measure precise azimuth displacements using MAI rather than the pixel offset tracking method. The MAI technique utilizes azimuth split-beam InSAR processing to create a MAI interferogram from the two different-looking geometries [22], [23]. MAI represents a significant improvement in measuring along-track displacement compared to the pixel offset tracking method [24]. A theoretical analysis of the standard deviation of the ionospheric phase measurement from MAI is discussed and compared with theoretical standard deviations from other methods. A test of the proposed method on an ALOS PALSAR interferogram is followed by a regression analysis between the ionosphere-corrected interferometric phase and topographic height and a 2-D Fourier spectral analysis. In this test, comparison with other methods is not performed because other methods are not good enough to correct the ionospheric effect from an ALOS PALSAR interferogram.

\section{Methods}

Because the ionospheric phase distortion on SAR interferogram depends on the ionospheric TEC and the inverse of the radar frequency [10], [11], high TEC and low radar frequency cause significant ionospheric artifacts. The effect causes range and azimuth pixel displacements on a SAR image as well as phase distortion. We investigate the relationship between the azimuth offset and the interferometric phase as well as the relationship between the azimuth offset and the MAI phase. In this section, we define the relationship between the ionosphere- distorted interferometric phase and MAI phase and propose an efficient method to correct ionosphere-distorted interferograms.

\section{A. Ionospheric Effects and MAI Phase}

The ionosphere-distorted interferometric phase shift $\phi_{\mathrm{ION}}$ at an oblique angle is defined as [11], [25]

$$
\phi_{\mathrm{ION}}=-\frac{4 \pi K}{c f} \frac{1}{\cos \theta} \Delta \mathrm{TEC}
$$

where $K$ is a constant of $40.28\left(\mathrm{~m}^{3} / \mathrm{s}^{2}\right), c$ is the speed of light, $f$ is the radar frequency, $\theta$ is the incidence angle, and $\triangle$ TEC is the variation of TEC. The azimuth derivative of the ionospheric phase $d \phi_{\mathrm{ION}} / d x$ is related to the azimuth displacement as follows [11]:

$$
\frac{d \phi_{\mathrm{ION}}}{d x}=\alpha \frac{4 \pi}{\lambda} \Delta x
$$

where $x$ is the azimuth, $\Delta x$ is the azimuth displacement, $\lambda$ is the radar wavelength, and $\alpha$ is a system- and geometrydependent factor.

The azimuth displacement is also defined by the MAI phase

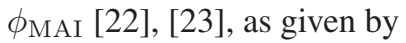

$$
\Delta x=-\frac{l}{4 \pi n} \cdot \phi_{\mathrm{MAI}}
$$

where $l$ is the effective antenna length and $n$ is a normalized squint that is a fraction of the full aperture width.

The relationship between the ionospheric phase azimuth derivative $d \phi_{\mathrm{ION}} / d x$ and the MAI phase $\phi_{\mathrm{MAI}}$ can be expressed as

$$
\frac{d \phi_{\mathrm{ION}}}{d x}=-\alpha \cdot \frac{l}{n \lambda} \cdot \phi_{\mathrm{MAI}} .
$$

Equation (4) indicates that the ionospheric phase derivative in azimuth is linearly proportional to the MAI phase. Consequently, the ionospheric phase is defined by the MAI phase as

$$
\phi_{\mathrm{ION}}=-\alpha \cdot \frac{l}{n \lambda} \cdot \int \phi_{\mathrm{MAI}} d x
$$

and the TEC variation is also given by

$$
\Delta \mathrm{TEC}=\frac{c f \cos \theta}{4 \pi K} \cdot \frac{\alpha l}{n \lambda} \cdot \int \phi_{\mathrm{MAI}} d x .
$$

This indicates that the ionospheric phase and the TEC variations can be estimated by integrating the MAI phase into the azimuth direction. Therefore, assuming the MAI phase entirely due to the ionosphere, we can measure and correct the ionospheric phase from a SAR interferogram using a MAI interferogram.

The measurement uncertainty can be derived from (4) as follows:

$$
\sigma_{\phi, \mathrm{ION}}=\frac{\Delta_{\mathrm{az}}}{\sqrt{2}} \cdot \frac{l \cdot|\alpha|}{n \lambda} \cdot \sigma_{\phi, \mathrm{MAI}}
$$

where $\sigma_{\phi, \mathrm{MAI}}$ and $\sigma_{\phi, \mathrm{ION}}$ are the standard deviations of the MAI and the ionospheric phase, respectively, and $\Delta_{\mathrm{az}}$ is the multilooked azimuth pixel spacing of the interferogram. Equation (7) is derived by assuming that the ionospheric phases of two adjacent azimuth pixels on a radar interferogram are independent variables [25]. 
The standard deviation of the MAI phase $\phi_{\mathrm{MAI}}$ is defined as [23]

$$
\sigma_{\phi, \mathrm{MAI}} \approx \frac{1}{\sqrt{N_{L}}} \frac{\sqrt{1-\gamma^{2}}}{\gamma}
$$

where $\gamma$ is the total correlation and $N_{L}$ is the effective look number for MAI interferograms.

The standard deviation of the TEC variation $\sigma_{\triangle \mathrm{TEC}}$ is calculated as

$$
\sigma_{\Delta \mathrm{TEC}}=\frac{c f \cos \theta}{4 \pi K} \cdot \frac{\Delta_{\mathrm{az}}}{\sqrt{2}} \cdot \frac{l \cdot|\alpha|}{n \lambda} \cdot \sigma_{\phi, \mathrm{MAI}} .
$$

Finally, the measurement uncertainties of the TEC variation and the ionospheric phase depend on the uncertainty of the MAI phase.

\section{B. Ionospheric Correction}

From (4), it can be observed that the ionospheric phase azimuth derivative is a linear function of the MAI phase. For ALOS PALSAR, the achieved accuracy of the MAI is about $0.8 \%$ of the azimuth resolution and is twice as good as the result obtained from the pixel offset tracking method (about $1.6 \%$ of the azimuth resolution) [24]. The improvement of the MAI technique on the azimuth displacement measurement has been demonstrated in [4] and [22]-[24].

After the InSAR and MAI interferograms are created and coregistered, the relationship between the InSAR phase and the MAI phase can be derived from (4)

$$
\frac{\Delta \phi_{\mathrm{InSAR}}(x, r)}{\Delta_{\mathrm{az}}}=\alpha \cdot \bar{\phi}_{\mathrm{MAI}}(x, r)+\beta
$$

where $r$ is the range, $\beta$ is the offset value used to calculate the reference phase of the MAI interferogram, $\Delta \phi_{\mathrm{InSAR}} / \Delta_{\mathrm{az}}$ is the InSAR phase azimuth derivative, the InSAR phase difference $\Delta \phi_{\text {InSAR }}$ is

$$
\Delta \phi_{\operatorname{InSAR}}(x, r)=\phi_{\operatorname{InSAR}}(x+1, r)-\phi_{\operatorname{InSAR}}(x, r)
$$

and the scaled MAI phase $\bar{\phi}_{\mathrm{MAI}}$ is defined as

$$
\bar{\phi}_{\mathrm{MAI}}(x, r)=-\frac{l}{n \lambda} \cdot \phi_{\mathrm{MAI}}(x, r) .
$$

The parameters $\alpha$ and $\beta$ can be determined by a polynomial fit using all of the coherent pixels. To improve the efficiency of the parameter calculation, it is necessary to exclude any pixels containing large surface deformation. Once these parameters are estimated, we can calculate the ionospheric phase $\phi_{\mathrm{ION}}$ as

$$
\phi_{\mathrm{ION}}(x, r)=\sum_{u=1}^{x}\left[\left(\alpha \cdot \bar{\phi}_{\mathrm{MAI}}(u, r)+\beta\right) \cdot \Delta_{\mathrm{az}}\right]+C(r)
$$

where $C$ is the integral constant that varies along the range position. The integral constants are unknown because they are in the ionospheric phase at $x=0$. Therefore, while these constants cannot be determined directly, they can be estimated from the InSAR interferogram by assuming a correlation between an InSAR and an ionospheric phase. Finally, the corrected InSAR phase $\hat{\phi}_{\text {InSAR }}$ can be obtained by

$$
\hat{\phi}_{\mathrm{InSAR}}(x, r)=\phi_{\operatorname{InSAR}}(x, r)-\phi_{\mathrm{ION}}(x, r) .
$$
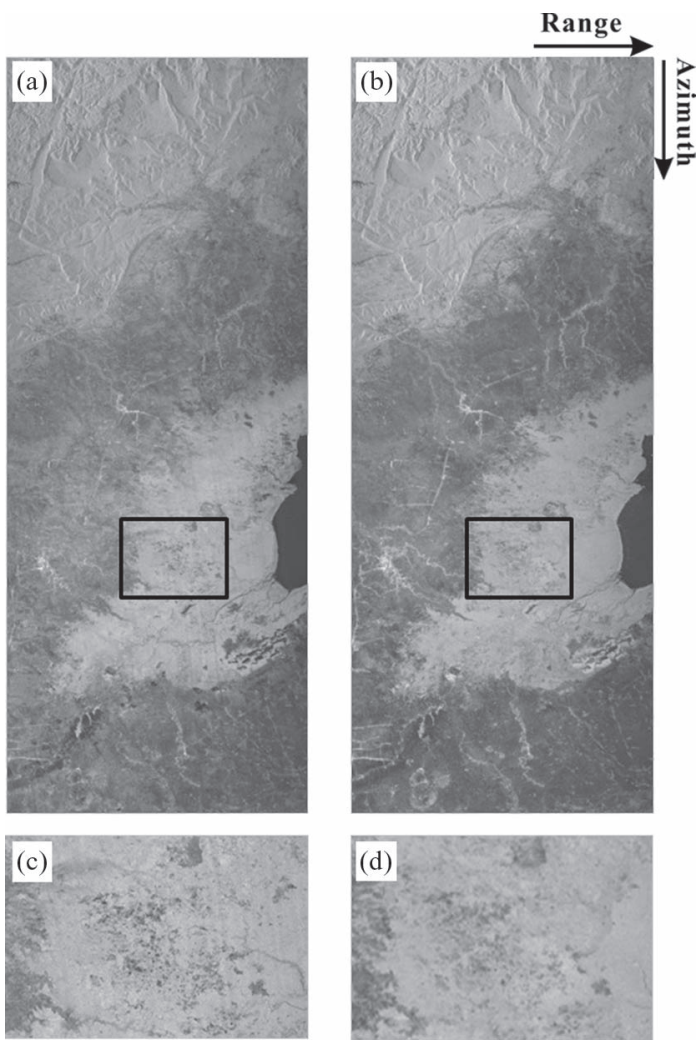

Fig. 1. Multilooked ALOS PALSAR images acquired on (a) January 17 and (b) March 3, 2008. (c) and (d) High-resolution images show over areas outlined by boxes in (a) and (b) that suffer from severe ionospheric distortions called azimuth streaking.

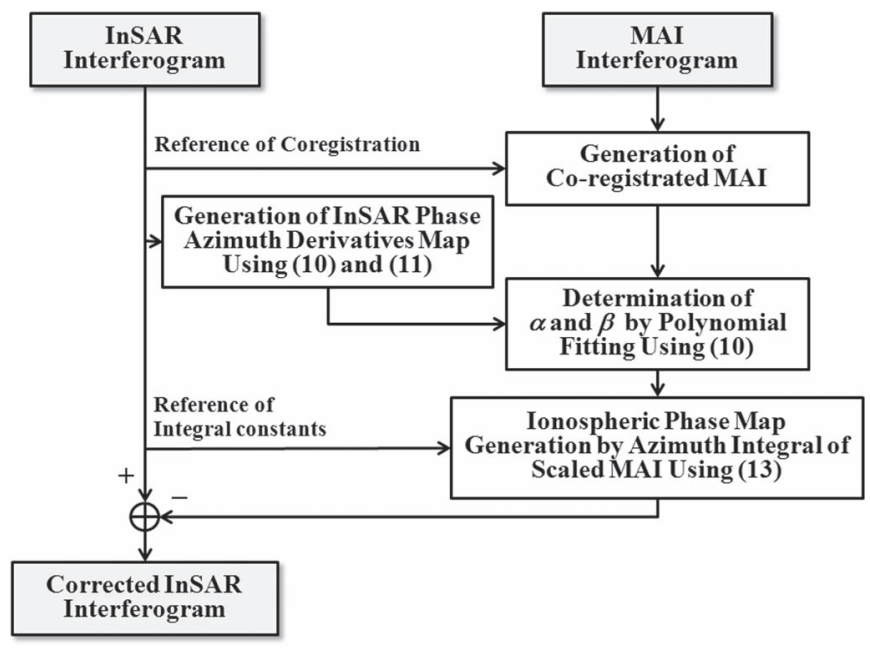

Fig. 2. Detailed processing flow of the proposed method.

\section{RESUltS}

\section{A. Test Data and Processing}

The performance of the proposed method was tested on an ALOS PALSAR interferometric pair acquired on January 17 [Fig. 1(a) and (c)] and March 3, 2008 [Fig. 1(b) and (d)]. The test site is located near Tonle Sap Basin, Cambodia. The January 17 image has strong ionospheric distortions causing shifts in azimuth pixel positions often referred to as azimuth streaking [Fig. 1(a) and (c)]. These data pairs are ideal to 
TABLE I

Characteristics of Interferometric Pair Used in This Study. ${ }^{1)} f_{\mathrm{DC}, f}, f_{\mathrm{DC}, c}$, AND $f_{\mathrm{DC}, b}$ Denote The Forward, Average, and Backward Doppler Centroids, and $\Delta f_{D, S}$ Is the Subaperture Processing Bandwidth. ${ }^{2)} B_{\perp}$ Is

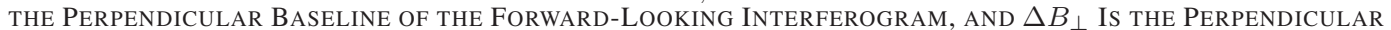
Baseline Difference Estimated by the Second-Order Polynomial Model Proposed in This Study

\begin{tabular}{c|c|c|c|c|c|c|c|c|c}
\hline \hline Master & Slave & $\begin{array}{c}\text { Beam } \\
\text { Mode }\end{array}$ & $\begin{array}{c}\text { Incidence } \\
\text { Angle (deg.) }\end{array}$ & $\begin{array}{c}\mathrm{f}_{\mathrm{DC}, \mathrm{f}} \mathrm{f}^{\mathrm{l}} \\
(\mathrm{Hz})\end{array}$ & $\begin{array}{c}\mathrm{f}_{\mathrm{DC}, \mathrm{c}}{ }^{1)} \\
(\mathrm{Hz})\end{array}$ & $\begin{array}{c}\left.\mathrm{f}_{\mathrm{DC}, \mathrm{b}}{ }^{\mathrm{l}}\right) \\
(\mathrm{Hz})\end{array}$ & $\begin{array}{c}\Delta \mathrm{f}_{\mathrm{D}, \mathrm{S}^{1}}{ }^{1} \\
(\mathrm{~Hz})\end{array}$ & $\begin{array}{c}\mathrm{B}_{\perp}{ }^{2)} \\
(\mathrm{m})\end{array}$ & $\begin{array}{c}\left.\Delta \mathrm{B}_{\perp}{ }^{2}\right) \\
(\mathrm{m})\end{array}$ \\
\hline $17 / 01 / 2008$ & $03 / 03 / 2008$ & FBS & 38.7 & 451.2 & 46.9 & -357.4 & 801.3 & 267.4 & 0.009 \\
\hline \hline
\end{tabular}

1) $f_{D C, f}, f_{D C, c}$ and $f_{D C, b}$ denote the forward, average, and backward Doppler Centroids, and $\Delta f_{D, S}$ is the sub-aperture processing bandwidth. ${ }^{2)} \mathrm{B}_{\perp}$ is the perpendicular baseline of the forward-looking interferogram, and $\Delta \mathrm{B}_{\perp}$ is the perpendicular baseline difference estimated by the second order polynomial model proposed in this study.
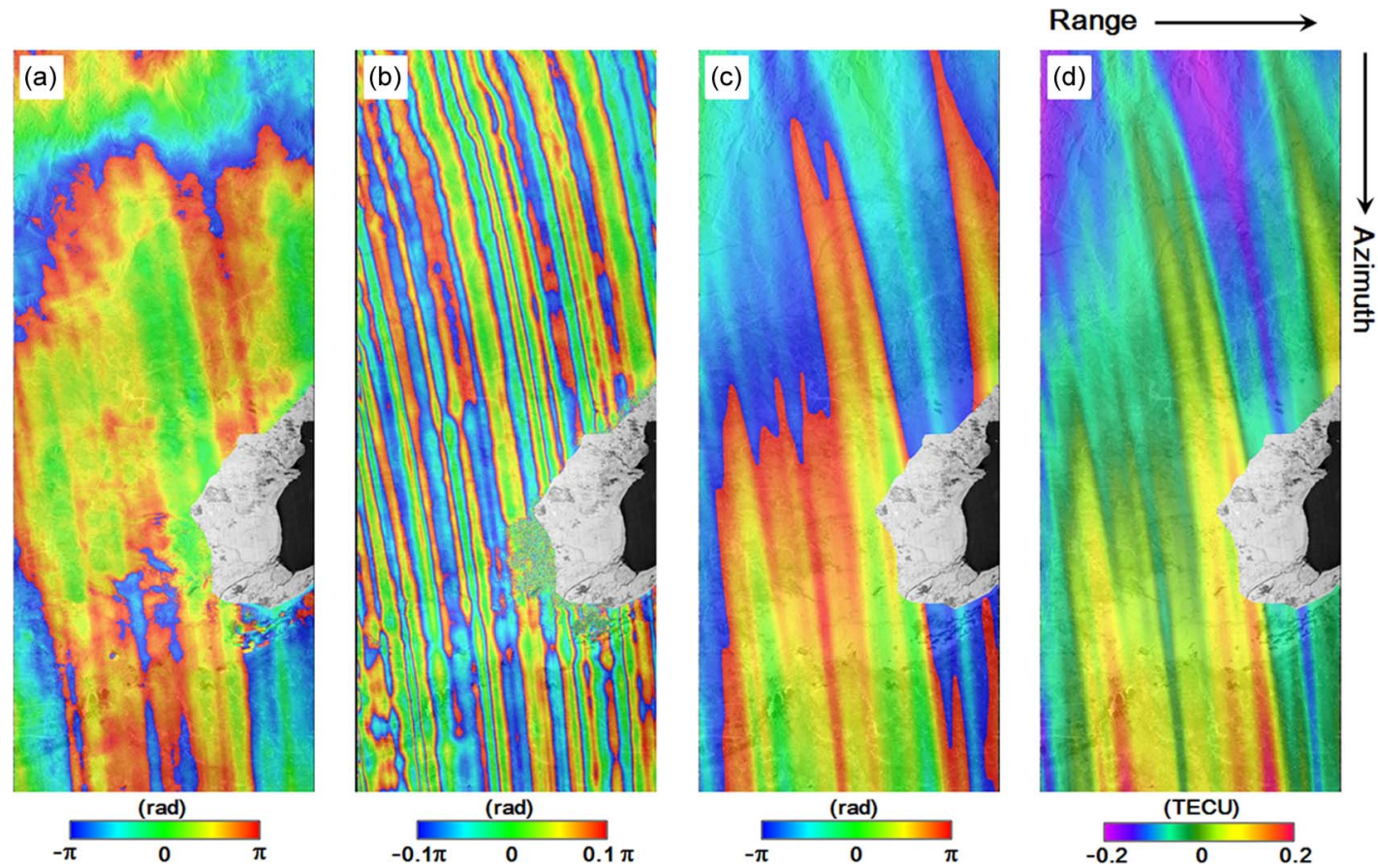

Fig. 3. (a) InSAR interferogram, (b) MAI interferogram, (c) ionospheric phase map, and (d) TEC variation map used to validate the experimental performance of the proposed method. Note that the one fringe in the MAI interferogram represents between 0 and $0.2 \pi$. The area of coherence loss is uncolored.

test the performance of the proposed method because of the following reasons: 1) Interferometric coherence is high due to the small perpendicular baseline (about $267 \mathrm{~m}$ ) and the short time separation (46 days), and 2) there is a strong ionospheric signal.

The detailed processing flow of the proposed method is summarized in Fig. 2. There are five processing steps: 1) creation and coregistration of an unwrapped InSAR and an unwrapped MAI interferogram; 2) estimation of an InSAR phase azimuth derivative $\left(\Delta \phi_{\text {InSAR }} / \Delta_{\text {az }}\right)$ map using $(10)$ and $\left.(11) ; 3\right)$ determination of $\alpha$ and $\beta$ from the ionospheric phase azimuth derivative map and the scaled MAI interferogram using (10); 4) generation of an ionospheric phase map from an azimuth integral of the scaled MAI interferogram using (13); and 5) generation of the corrected InSAR interferogram by subtracting the ionospheric phase map from the InSAR interferogram using (14).

The InSAR interferogram is created by a complex multilook operation of $20 \times 40$ looks $(\sim 110 \mathrm{~m} \times 125 \mathrm{~m})$ in range and azimuth directions. It is smoothed using an adaptive filter [26] with a window size of 32 to reduce phase variance. The multilook operation is applied using a two-step strategy as follows: 1) $4 \times 8$ looks before the flat-Earth and topographic phase corrections and 2) $5 \times 5$ looks after the corrections to reduce phase noise [23].

The MAI interferogram is created by Jung's MAI approach [23]. For the MAI processing of this interferometric pair, the forward, average, and backward Doppler centroids of 451.2, 46.9 , and $-357.4 \mathrm{~Hz}$, respectively, and the subaperture processing bandwidth of $801.3 \mathrm{~Hz}$ are calculated by MAI azimuth common band filtering [23]. A normalized squint of 0.5 and an effective antenna length of $8.9 \mathrm{~m}$, respectively, are used for this calculation. The MAI characteristics of this pair are summarized in Table I. Forward- and backward-looking singlelook-complex images are created from radar raw signal data, and then forward- and backward-looking interferograms are generated, and multilooked and smoothed by the same multilook factor and filter used for the InSAR processing. The final 


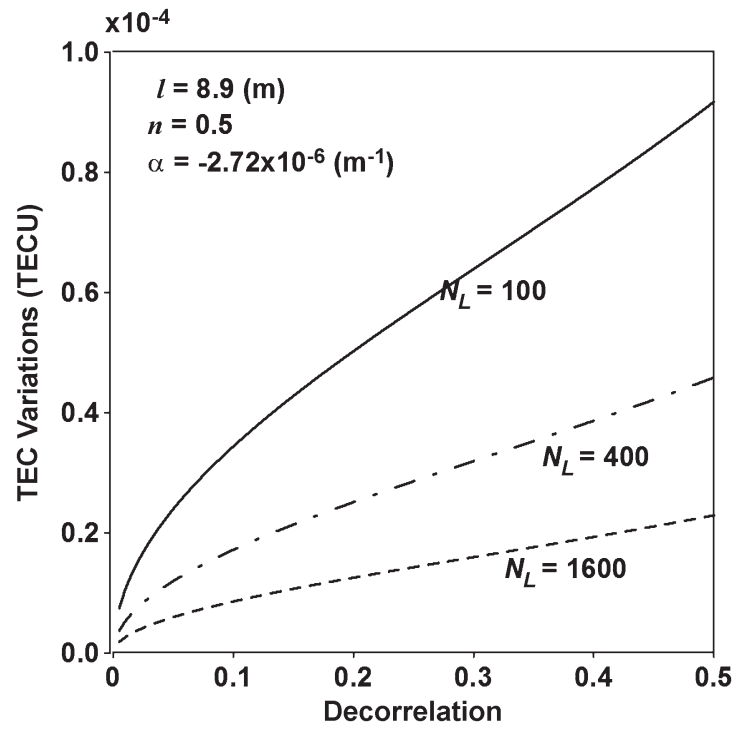

Fig. 4. Theoretical standard deviation of the TEC variation measurement. The ALOS PALSAR system parameters $f=1.270 \mathrm{GHz}$ and $\mathrm{PRF}=2160 \mathrm{~Hz}$ and the values $l=8.9 \mathrm{~m}, n=0.5, \alpha=-2.72 \times 10^{-6} \mathrm{~m}^{-1}$, and $N_{L}=100$, 400 , and 1600 are used for this theoretical analysis. The parameter $\alpha$ is estimated from the real interferometric pair used in this study.

MAI interferogram is generated after residual flat-Earth and topographic phase corrections are applied [23].

After the InSAR and MAI interferograms are created, coregistration can be accomplished by simple translation as they have the same relative geometry. Fig. 3(a) and Fig. 3(b) show the ionosphere-distorted InSAR and MAI interferograms, respectively. As shown in Fig. 3(a) and (b), the azimuth streaks on the InSAR and MAI interferograms are oblique to the range direction. The positive and negative maxima of the MAI phase are about $\pm 0.85 \mathrm{rad}$, corresponding to along-track displacements of about $\pm 1.2 \mathrm{~m}$.

\section{B. Theoretical Analysis}

A theoretical standard deviation of the TEC measurement is shown in Fig. 4. The ALOS PALSAR system parameters $f=1.270 \mathrm{GHz}$ and $\mathrm{PRF}=2160 \mathrm{~Hz}$ and the values $l=8.9 \mathrm{~m}$, $n=0.5, \alpha=-2.72 \times 10^{-6} \mathrm{~m}^{-1}$, and $N_{L}=100,400$, and 1600 are used for this theoretical analysis. The parameter $\alpha$ is estimated from the interferometric pair used in this study. The look numbers $N_{L}$ of 100,400 , and 1600 are determined by average window sizes of $10 \times 20,20 \times 40$, and $40 \times$ 80 pixels, respectively. As shown in Fig. 4, the standard deviation of the TEC variations rapidly increases as the total decorrelation increases. When correlation $\gamma$ is 0.9 , the standard deviations of the TEC variations $\left(\sigma_{\triangle \mathrm{TEC}}\right)$ are approximately $3.4 \times 10^{-5}, 1.7 \times 10^{-5}$, and $8.6 \times 10^{-6}$ TEC units (TECU) for $N_{L}=100,400$, and 1600 , respectively. Assuming a moderate interferometric coherence of $\gamma=0.5$, the standard deviations of the TEC variations are less than $1.0 \times 10^{-4}$ TECU for $N_{L}=100,400$, and 1600 . Considering that the accuracy requirements in ionospheric correction are about $0.22 \mathrm{TECU}$ for topographic mapping and about 0.007 TECU for deformation monitoring [25], our result indicates that the performance of ionospheric correction by the proposed method largely exceeds the required accuracy. An accuracy of 0.1 TECU for ALOS

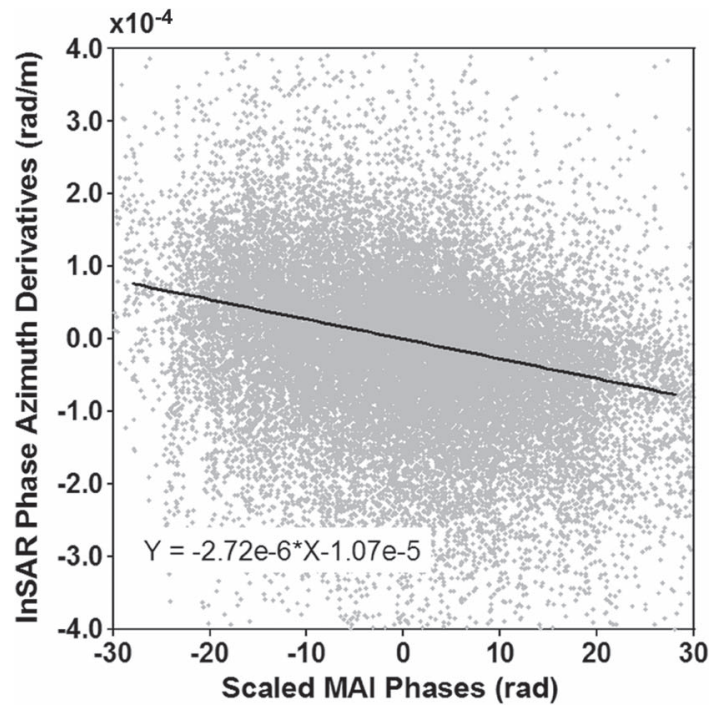

Fig. 5. Variations of the InSAR phase azimuth derivative with respect to the scaled MAI phase.

PALSAR fine-beam single-polarization images can be achieved using the range group-phase delay difference and the range split-spectrum methods for $N_{L}=4000$ and a correlation of $\gamma=0.5$ [11], [14]. Therefore, the proposed method meets the required accuracy of ionospheric correction for topographic mapping and deformation monitoring and is also superior to other ionosphere correction methods, assuming no deformation in azimuth.

\section{Results of Ionospheric Correction}

After the InSAR and MAI interferograms are created and coregistered, the azimuth derivatives of the InSAR phase $\Delta \phi_{\text {InSAR }} / \Delta_{\text {az }}$ are calculated. The parameters $\alpha$ and $\beta$ in (10) are estimated by polynomial fitting between the azimuth derivative of the InSAR phase and the scaled MAI phase defined by (12). Fig. 5 shows the variations of the InSAR phase azimuth derivative with respect to the scaled MAI phase. While the fluctuations in azimuth derivative of the InSAR phase are large, the fluctuations of the scaled MAI phase are small. This difference is due to the lower precision of the InSAR phase azimuth derivative and the higher precision of the scaled MAI phase. To overcome the low precision of the InSAR phase derivative in azimuth, an iterative approach is used to estimate the parameters $\alpha$ and $\beta$. These parameters are calculated by first-order polynomial fitting and updated by removing outliers using the conventional significance level of 0.05 . The updates continue until outliers do not exist. For this ALOS pair, the parameters $\alpha$ and $\beta$ are estimated as $-2.72 \times 10^{-6} \mathrm{~m}^{-1}$ and $-1.07 \times 10^{-5} \mathrm{rad}$, respectively. Once these parameters are obtained, the ionospheric phase derivative map can be generated from the scaled MAI phase if large azimuth displacements due to ground deformation are negligible.

The ionospheric phase map is generated by the azimuth integration of the azimuth derivative of the ionospheric phase using (13) [Fig. 3(c)]. This integration is implemented by the accumulation of the ionospheric phase derivative along the azimuth presented in (13) and requires an efficient method 


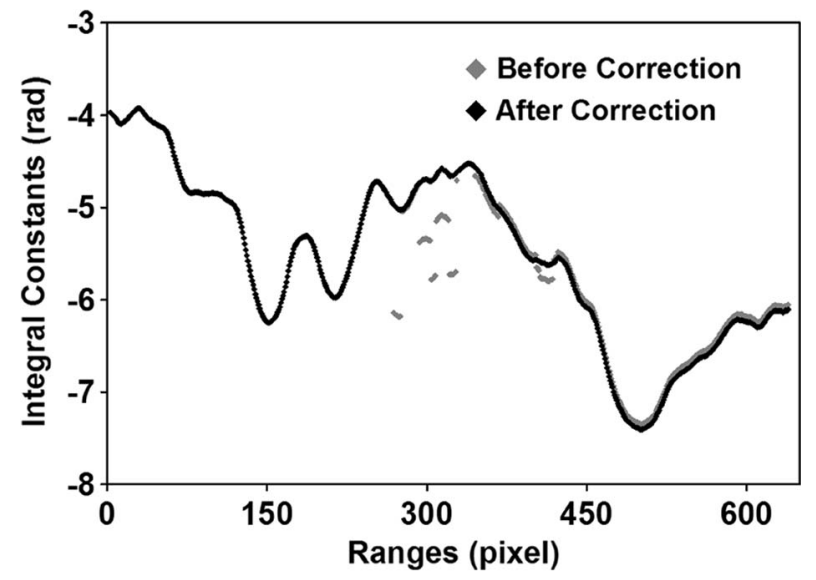

Fig. 6. Integral constants (gray diamonds) before and (black diamonds) after the correction of discontinuities.

to estimate the integral constants that are a function of range location. The azimuthal accumulation map is first generated by the summation of the ionospheric phase derivative in azimuth without considering integral constants, and a difference map is generated by subtracting the azimuthal accumulation map from the InSAR image.

The integral constants can be estimated by averaging the difference map in azimuth direction, because the correlation between the InSAR and the ionospheric phase can be assumed. For more efficient estimation, the aforementioned iterative tactic used for the parameter estimation is applied to the determination of the integral constants. This calculation largely depends on the correlation between the ionospheric and the InSAR phase, which can be compromised by the tropospheric artifact, topographic height error, surface deformation, etc. Thus, the integral constants can be distorted if the correlation is not high enough and can have the discontinuities if the correlation difference between adjacent azimuth lines is high. The integral constants estimated by this approach are represented by the solid gray diamonds in Fig. 6 . The integral constants include some discontinuities due to the high correlation difference. These discontinuities can cause azimuth stripes on the ionospheric phase map if they are not corrected. In this paper, the stripes are detected from the range gradients of the ionospheric phase map using the approach proposed in [27]. The integral constants are corrected using the adjacent integral constants. The corrected integral constants are shown as the black diamonds in Fig. 6.

The final ionospheric phase map is generated by the azimuthal integration of the ionospheric phase azimuth derivative using corrected integral constants [Fig. 3(c)]. This map has wave patterns that are oblique to the range direction and is converted into the TEC variation map using (1) [Fig. 3(d)]. The positive and negative maxima of the TEC variations are about \pm 0.2 TECU [Fig. 3(d)]. As previously mentioned, an accuracy of 0.1 TECU requires a multilook number of about 4000 for the range group-phase delay difference and the range split-spectrum methods. Thus, for the PALSAR system with the properties of this interferogram, the MAI technique yields more accurate results at a finer resolution compared with other methods.
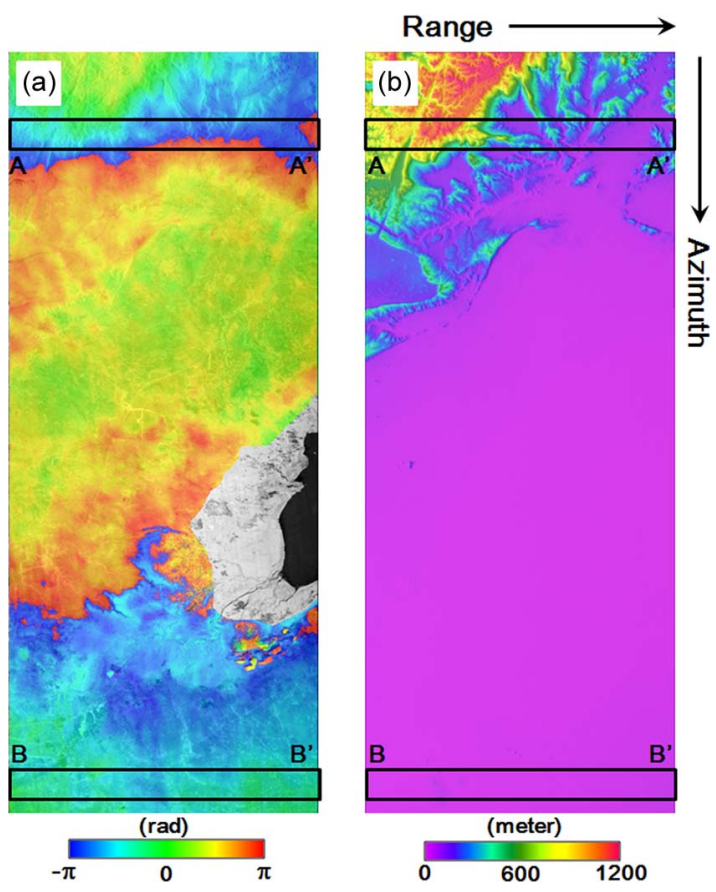

Fig. 7. (a) Corrected InSAR interferogram and (b) the corresponding colored shaded relief map.

Fig. 7 shows the corrected InSAR interferogram and the colored shaded relief map. As shown in Fig. 7(a), the ionospheric wave patterns that are oblique to the range direction have been removed, and a topography-dependent phase pattern is visible. The topography-dependent pattern is a tropospheric artifact that is often closely related to topographic height [28]. Thus, for a quantitative validation of the proposed method, we compare the global Shuttle Radar Topography Mission digital elevation model [Fig. 7(b)] with the uncorrected and corrected InSAR interferograms.

Fig. 8 shows the variations of the uncorrected and corrected InSAR phases with respect to topographic height over two representative areas marked with $A-A^{\prime}$ and $B-B^{\prime}$. The uncorrected InSAR phase is more scattered than the corrected phase (Fig. 8). In $B-B^{\prime}$, the uncorrected InSAR phase ranges from about -8 to $-2 \mathrm{rad}$, while the corrected phase is between about 1 and 3 rad. The root-mean-square errors (rmses) for the regression results reduce from 0.80 to $0.36 \mathrm{rad}$ for $A-A^{\prime}$ and from 1.46 to $0.34 \mathrm{rad}$ for $B-B^{\prime}$. This reduction in dynamic range suggests suppression of the ionospheric component.

A 2-D Fourier analysis also illustrates the reduction of wave energy from the interferogram (Fig. 9). Fig. 9(a), (b), and (c) shows the 2-D power spectra of the uncorrected and corrected interferograms and ionospheric phase map, respectively. To generate these power spectra, a Hanning window is applied to the data, as well as zero-mean normalization and zero padding, prior to 2-D Fourier transformation. An oblique line [Fig. 9(a)] that is normal to the ionospheric wave pattern shown in Fig. 3(a) characterizes the power spectrum of the uncorrected interferogram. However, this oblique line is absent in the corrected power spectrum [Fig. 9(b)], indicating that the corrected InSAR interferogram does not include the ionospheric wave pattern. The power spectrum of the ionospheric phase map is characterized by the same oblique line as the ionospheric artifact, as well as a horizontal line [Fig. 9(c)]. 


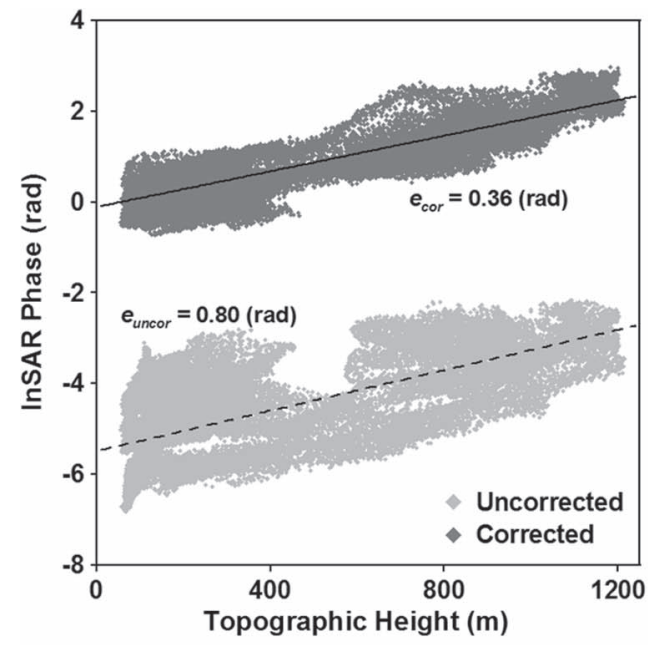

(a)

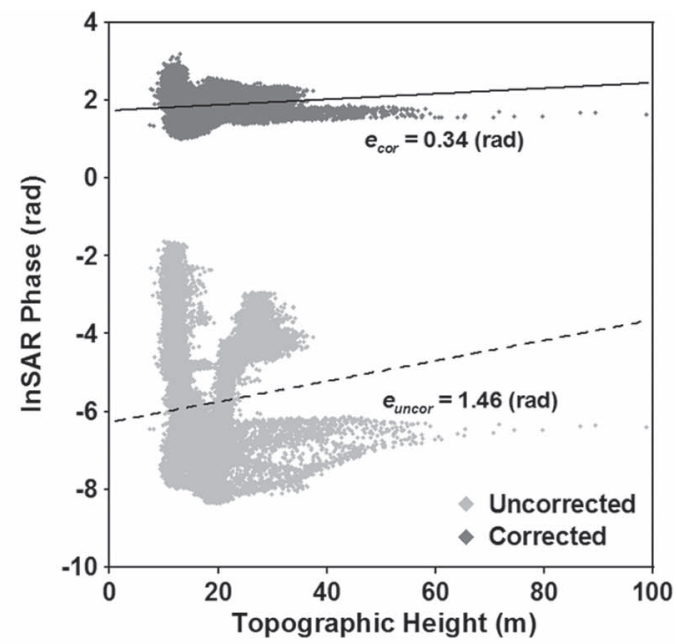

(b)

Fig. 8. Variations of the uncorrected and corrected InSAR phases with respect to the topographic height along (a) profile $A-A^{\prime}$ and (b) profile $B-B^{\prime}$ as shown in Fig. 7. The gray and black solid diamonds represent the uncorrected and corrected InSAR phases, respectively. The dashed and solid lines show the linear regression results of the uncorrected and corrected phases in the profiles $A-A^{\prime}$ and $B-B^{\prime}$, respectively.
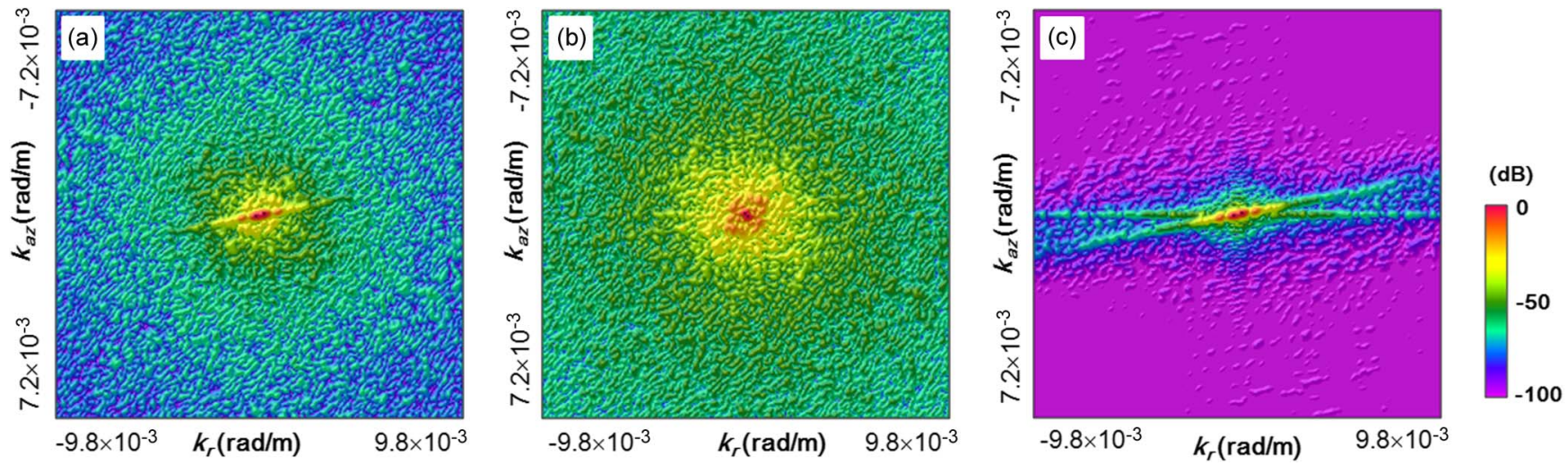

Fig. 9. Two-dimensional power spectra of (a) uncorrected interferogram, (b) corrected interferogram, and (c) ionospheric phase map. $k_{\text {az }}$ and $k_{r}$ denote the azimuth and range wavenumbers, respectively.

The horizontal line is parallel to the range direction and is caused by subtle azimuth integral artifacts [Fig. 9(c)]. However, this line is not present in the power spectrum of the corrected interferogram because its magnitude is much smaller than that of the oblique line caused by the ionosphere artifact. Thus, the azimuth integral artifact can be ignored in the corrected InSAR interferogram.

Regression and the 2-D Fourier spectral analyses demonstrate that the proposed method can extract a precise ionospheric phase map and correct the InSAR interferogram. Therefore, the proposed method can effectively correct strong ionospheric phase distortions in the L-band SAR systems such as ALOS PALSAR or other low-frequency SAR systems.

\section{CONCLUSION}

In this paper, we have presented an efficient method to measure and correct ionospheric phase artifacts in an InSAR image using the MAI interferogram. We have also evaluated the theoretical and experimental performances of the proposed method. Our ionospheric correction processing procedure includes the following steps: 1) creation and coregistration of an
InSAR and a MAI interferogram; 2) estimation of an azimuth derivative map of the InSAR phase; 3) determination of $\alpha$ and $\beta$ parameters; 4) generation of ionospheric phase screen; and 5) creation of the corrected InSAR interferogram. Based on our theoretical analysis, the standard deviation of TEC variations is less than $1.0 \times 10^{-4} \mathrm{TECU}$ for $N_{L}=100,400$, and 1600 , when a moderate interferometric coherence of $\gamma=0.5$ is considered. These results indicate that the proposed method can map the TEC variation at an accuracy that far exceeds the required accuracy for topographic mapping (0.22 TECU) or deformation monitoring (0.007 TECU). Experimental tests on an ALOS PALSAR interferometric pairs were conducted by a regression analysis of the ionosphere-corrected interferometric phase and topographic height as well as a 2-D Fourier spectral analysis. In the regression analysis, the rmse is improved by a factor of two to four after the ionosphere correction. In the 2-D Fourier spectral analysis, an oblique line that is normal to the ionospheric wave pattern in the power spectrum of uncorrected interferogram is absent in the power spectrum of the corrected image. These results demonstrate that the proposed method can be effectively applied to correct ionospheric phase distortions in InSAR images. 


\section{REFERENCES}

[1] J. Biggs, F. Amelung, N. Gourmelen, T. H. Dixon, and S.-W. Kim, "InSAR observations of 2007 Tanzania rifting episode reveal mixed fault and dyke extension in an immature continental rift," Geophy. J. Int., vol. 179, no. 1 , pp. 549-558, 2009.

[2] M. Wei, D. Sandwell, Y. Fialko, and R. Bilham, "Slip on faults in the Imperial Valley triggered by the 4 April $2010 \mathrm{Mw} 7.2$ El Mayor-Cucapah earthquake revealed by InSAR," Geophy. Res. Lett., vol. 38, no. L1, pp. L01308-1-L01308-6, 2011.

[3] Z. Lu, D. Dzurisin, J. Biggs, C. Wicks, Jr., and S. McNutt, "Ground surface deformation patterns, magma supply, and magma storage at Okmok volcano, Alaska, inferred from InSAR analysis: 1. Inter-eruptive deformation, 1997-2008," J. Geophys. Res., vol. 115, pp. В00B03-1-B00B03-14, 2011.

[4] H.-S. Jung, Z. Lu, J. S. Won, M. P. Poland, and A. Miklius, "Mapping three-dimensional surface deformation by combining multiple-aperture interferometry and conventional interferometry: Application to the June 2007 eruption of Kilauea volcano, Hawaii," IEEE Geosci. Remote Sens. Lett., vol. 8, no. 1, pp. 34-38, Jan. 2011.

[5] L. Gray, "Using multiple RADARSAT InSAR pairs to estimate a full three-dimensional solution for glacial ice movement," Geophy. Res. Lett., vol. 38, no. L5, pp. L05502-1-L05502-6, 2011.

[6] M. McMillan, A. Shepherd, N. Gourmelen, J.-W. Park, P. Nienow, E. Rinne, and A. Leeson, "Mapping ice-shelf flow with interferometric synthetic aperture radar stacking," J. Glaciol., vol. 58, no. 208, pp. 265277, Apr. 2012.

[7] J.-K. Choi, J.-S. Won, S. Lee, S.-W. Kim, K.-D. Kim, and H.-S. Jung, "Integration of GIS and SAR interferometry for a coal mine subsidence hazard map in Taebaek, Korea," Int. J. Remote Sens., vol. 32, no. 23, pp. 8161-8181, Dec. 2011.

[8] L. Zhang, Z. Lu, X. Ding, H.-S. Jung, G. Feng, and C.-W. Lee, "Mapping ground surface deformation using temporarily coherent point SAR interferometry: Application to Los Angeles Basin," Remote Sens. Environ., vol. 117, pp. 429-439, Feb. 2012.

[9] Z. Lu and O. Kwoun, "Radarsat-1 and ERS InSAR analysis over Southeastern Coastal Louisiana: Implications for mapping water-level changes beneath swamp forests," IEEE Trans. Geosci. Remote Sens., vol. 46, no. 8, pp. 2167-2184, Aug. 2008.

[10] J. E. Titheridge, "Determination of ionospheric electron content from the Faraday rotation of geostationary satellite signals," Planet. Space Sci., vol. 20, no. 3, pp. 353-369, Mar. 1972.

[11] F. Meyer, R. Bamler, N. Jakowski, and T. Fritz, "The potential of low-frequency SAR systems for mapping ionospheric TEC distributions," IEEE Geosci. Remote Sens. Lett., vol. 3, no. 4, pp. 560-564, Oct. 2006.

[12] D. Raucoules and M. de Michele, "Assessing ionospheric influence on L-band SAR data: Implications on coseismic displacement measurements of the 2008 Sichuan earthquake," IEEE Geosci. Remote Sens. Lett., vol. 7, no. 2, pp. 286-290, Apr. 2010.

[13] A. L. Gray, K. E. Mattar, and G. Sofko, "Influence of ionospheric electron density fluctuations on satellite radar interferometry," Geophy. Res. Lett., vol. 27, no. 10, pp. 1451-1454, 2000.

[14] R. Brcic, A. Parizzi, M. Eineder, R. Bamler, and F. Meyer, "Estimation and compensation of ionospheric delay for SAR interferometry," in Proc. IGARSS, Jul. 2010, pp. 2908-2911.

[15] E. J. M. Rignot, "Effect of Faraday rotation on L-band interferometric and polarimetric synthetic-aperture radar data," IEEE Trans. Geosci. Remote Sens., vol. 38, no. 1, pp. 383-390, Jan. 2000.

[16] P. A. Wright, S. Quegan, N. S. Wheadon, and C. D. Hall, "Faraday rotation effects on L-band spaceborne SAR data," IEEE Trans. Geosci. Remote Sens., vol. 41, no. 12, pp. 2735-2744, Dec. 2003.

[17] F. Meyer and J. B. Nicoll, "Prediction, detection, and correction of Faraday rotation in full-polarimetric L-band SAR data," IEEE Trans. Geosci. Remote Sens., vol. 46, no. 10, pp. 3076-3086, Oct. 2008.

[18] X. Pi, A. Freeman, B. Chapman, P. Rosen, and Z. Li, "Imaging ionospheric inhomogeneities using spaceborne synthetic aperture radar," J. Geophys. Res., vol. 116, no. A4, pp. A04303-1-A04303-13, 2011.

[19] R. Bamler and M. Eineder, "Accuracy of differential shift estimation by correlation and split-bandwidth interferometry for wideband and delta-k SAR systems," IEEE Geosci. Remote Sens. Lett., vol. 2, no. 2, pp. 151155, Apr. 2005.

[20] R. Brcic, M. Eineder, and R. Bamler, "Interferometric absolute phase determination with TerraSAR-X wideband SAR data," in Proc. Radar Conf., May 2009, pp. 1-6.

[21] P. A. Rosen, S. Hensley, and C. Chen, "Measurement and mitigation of the ionospheric in L-band interferometric SAR data," in Proc. Radar Conf., May 2010, pp. 1459-1463.
[22] N. B. D. Bechor and H. A. Zebker, "Measuring two-dimensional movements using a single InSAR pair," Geophy. Res. Lett., vol. 33, no. L16, pp. L16311-1-L16311-5, 2006.

[23] H.-S. Jung, J.-S. Won, and S.-W. Kim, "An improvement of the performance of multiple-aperture SAR interferometry (MAI)," IEEE Trans. Geosci. Remote Sens., vol. 47, no. 8, pp. 2859-2869, Aug. 2009.

[24] H.-S. Jung, Z. Lu, and L. Zhang, "Feasibility of along-track displacement measurement from Sentinel-1 interferometric wide-swath mode," IEEE Trans. Geosci. Remote Sens., vol. 51, no. 1, pp. 573-578, Jan. 2013.

[25] F. Meyer, "Performance requirements for ionospheric correction of lowfrequency SAR data," IEEE Trans. Geosci. Remote Sens., vol. 49, no. 10, pp. 3694-3702, Oct. 2011.

[26] R. M. Goldstein and C. L. Werner, "Radar interferogram filtering for geophysical applications," Geophys. Res. Lett., vol. 25, no. 21, pp. 40354038, 1998.

[27] H.-S. Jung, J.-S. Won, M.-H. Kang, and Y.-W. Lee, "Detection and restoration of defective lines in the SPOT 4 SWIR band," IEEE Trans. Image Process., vol. 19, no. 8, pp. 2143-2156, Aug. 2010.

[28] H. A. Zebker, P. A. Rosen, and S. Hensley, "Atmospheric effects in interferometric synthetic aperture radar surface deformation and topographic maps," J. Geophys. Res., vol. 102, no. B4, pp. 7547-7563, 1997.

[29] J. Chen and H. A. Zebker, "Ionospheric artifacts in simultaneous L-band ionospheric artifacts in simultaneous L-band InSAR and GPS observations," IEEE Trans. Geosci. Remote Sens., vol. 50, no. 4, pp. 1227-1239, Apr. 2012.

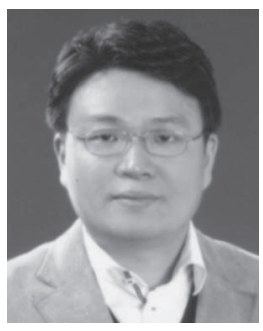

Hyung-Sup Jung (M'09) received the M.S. and Ph.D. degrees in geophysics and remote sensing from Yonsei University, Seoul, Korea, in 1998 and 2007 , respectively.

$\mathrm{He}$ is currently an Assistant Professor with the Department of Geoinfomatics, University of Seoul, Seoul. His primary research interests include developments of synthetic aperture radar (SAR), interferometric SAR (InSAR), multiple-aperture InSAR (MAI) and small baseline subset InSAR processors, and algorithms related to 3-D deformation mapping by combining MAI and InSAR and 2-D surface velocity estimation by combining MAI and along-track interferometry.

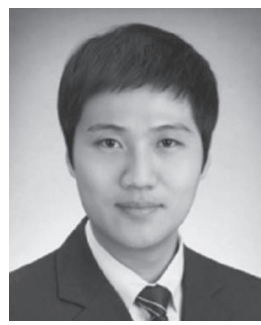

Dong-Taek Lee (S'11) received the B.S. and M.S. degrees in geoinformatics and remote sensing from the University of Seoul, Seoul, Korea, in 2010 and 2012, respectively.

He has investigated interferometric radar altimeter systems and ionospheric effect corrections of synthetic aperture radar (SAR) interferograms using multiple-aperture interferometry. He is currently a National Public Official with the Division of Geospatial Imagery Information and Photogrammetry, National Geographic Information Institute, Suwon, Korea. His research interests include remote sensing, aerial photogrammetry, SAR, and interferometric SAR. 


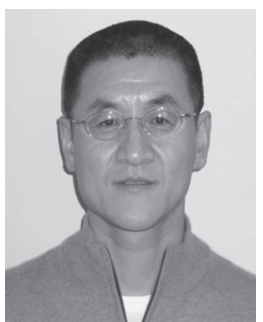

Zhong Lu (S'96-A'97-M'97-SM'07) received the M.S. degree from Beijing University, Beijing, China, in 1992 and the Ph.D. degree from the University of Alaska Fairbanks, Fairbanks, in 1996.

$\mathrm{He}$ is currently a Physical Scientist from Cascades Volcano Observatory, U.S. Geological Survey (USGS), Vancouver, WA. He is a Principal Investigator of projects funded by the National Aeronautics and Space Administration (NASA), European Space Agency, Japan Aerospace Exploration Agency, German Space Agency, and USGS on the study of land surface deformation using satellite interferometric synthetic aperture radar (InSAR) imagery. $\mathrm{He}$ is a Committee Member of the International User Team for Radarsat-C SAR Constellations, the GeoEarthscope InSAR User Working Group, the NASA Alaska Satellite Facility User Working Group, and NASA DESDynI-R Science Definition Team. He has produced more than 40 lead-authored and 60 coauthored peer-reviewed journal articles and book chapters focused on InSAR techniques and applications. His research interests include technique developments of SAR, InSAR, persistent scatterer InSAR processing, and their applications on natural hazard monitoring and natural resource management.

Dr. Lu was the recipient of an American Society for Photogrammetry and Remote Sensing Award for Best Scientific Paper in Remote Sensing, NASA Group Achievement Award, NASA Certificate of Appreciation, Raytheon Distinguished Level Award for Excellence in Technology, Science Applications International Corporation (SAIC) Technical Fellow, and Jerald Cook Memorial Award.

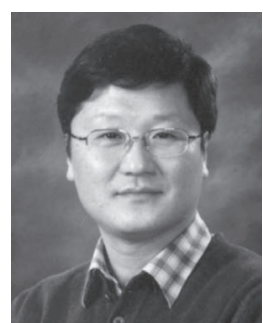

Joong-Sun Won (S'92-M'93-SM'03) received the B.S. and M.S. degrees in geology and geophysics from Yonsei University, Seoul, Korea, in 1983 and 1985 , respectively, and the Ph.D. degree in geophysics from the University of Manitoba, Winnipeg, MB, Canada, in 1993.

From 1993 to 1996 , he was a Senior Scientist with the Marine Geology Division, Korea Ocean Research and Development Institute. He is currently a Professor with the Department of Earth System Sciences, Yonsei University. His research interests include remote sensing, tidal flats, geohazards, signal processing, radar interferometry, and velocity retrieval from synthetic aperture radar data. 